\title{
Penile Size Dissatisfaction
}

\author{
Hester Pastoor, MSc, and Angela Gregory, BA, PGDip ${ }^{2}$
}

\begin{abstract}
Background: Men concerned about their penis size often consult professionals working in urology, andrology, surgery, and sexual medicine.

Aim: To inform professionals in the sexual medicine field about small penis syndrome as a clinical syndrome and to provide recommendations for treatment.

Methods: This was an overview of the existing literature combined with our extensive clinical experience.

Results: Small penis syndrome is a syndrome with psychiatric comorbidities and social consequences that impair life. Men with these concerns tend to be susceptible for treatment that is not evidence based and potentially harmful.

Clinical Implications: Treatment of men with concerns about penis size should start with a thorough biopsychosocial assessment, followed by extensive psychoeducation, counselling, and psychological interventions, even if surgery is being considered.

Strengths \& Limitations: The strength of this study is the concise overview of the existing literature combined with clinical experience which leads to important recommendations. Limitation is that this is not a systematic review.
\end{abstract}

Conclusion: Complaints about penis size should be taken seriously, and a thorough biopsychosocial and multidisciplinary assessment is required.

Copyright (C) 2020, International Society for Sexual Medicine. Published by Elsevier Inc. All rights reserved.

Key Words: Penile Size; Small Penis Syndrome; Body Dysmorphic Disorder

John (28 years) sees an urologist requesting for surgical penile enhancement, as he is dissatisfied with his flaccid penile length. As a 12-year-old boy, he was bullied by other boys during communal showers about his penile size. In addition, when he looked down at his own penis, it seemed really small compared with that of the other boys. He is gay, and has never had a relationship or any sexual experience with a partner. He is convinced that other gay men will find his penis too small, and he will not be desirable as a sexual partner. His beliefs are confirmed by gay pornography and information on the internet about what is the "normal" size of a penis. However, he has never had the courage to measure himself.

Received September 4, 2019. Accepted March 29, 2020.

'Registered Psychotherapist, Registered Sexologist, ECPS, PhD Student, Division of Reproductive Medicine, Department of Obstetrics and Gynecology, Erasmus MC, University Medical Center, Rotterdam, The Netherlands;

${ }^{2}$ COSRT Accredited Psychosexual Therapist, Division of Family Health, Department of Sexual Health, Chandos Clinic, Nottingham University Hospital Trust, Nottingham, United Kingdom

Copyright (๑) 2020, International Society for Sexual Medicine. Published by Elsevier Inc. All rights reserved.

https://doi.org/10.1016/j.jsxm.2020.03.015
In 1989, Murtagh ${ }^{1}$ published "The small penis syndrome" (SPS). He described the concerns and preoccupations men have about the size of their penis and its consequences on sexual performance. Almost 20 years later, Wylie and Eardley ${ }^{2}$ gave a thorough overview of the "syndrome," its definition, prevalence, assessment, psychosocial comorbidities, and treatment options. During more than 20 years of clinical experience, we have seen many men who present with SPS. In this invited commentary, we describe our experiences with assessment and treatment of SPS. In addition, we provide recommendations for clinical practice. With this commentary, we aim to inform professionals in the sexual medicine field about the SPS as a clinical syndrome and to provide recommendations for treatment. Case examples illustrate the text.

SPS can be defined as "an anxiety about the genitals being observed, directly or indirectly (when clothed), because of concern that the flaccid penis length and/or its girth is less than the normal for an adult male, despite evidence from a clinical examination to counter this." In general, men who present with SPS have a normal-sized penis. ${ }^{2-4}$ Men with SPS and sexual difficulties, such as erectile dysfunction, loss of sexual interest, or avoidance of sexual relationships, had not necessarily been examined physically. In addition, concerns regarding penis size were only identified during sexual history taking. Not all men 
concerned with penis size measure themselves. In addition, their ideal penis size is often highly discrepant from their actual penis size, ${ }^{5}$ which they often underestimate. ${ }^{3}$

\section{EPIDEMIOLOGY, DEVELOPMENT, AND PRESENTATION}

The extent of the problem is not well known. However, in a large Internet-based survey of 52,031 heterosexual men, $12 \%$ rated their penis as small, $22 \%$ as large, and $66 \%$ as average. ${ }^{6}$ What is a normal penis size? A systematic review of $15,000 \mathrm{men}^{7}$ found that the average flaccid length was 3.6 inches $(9.16 \mathrm{~cm}$; 5 th percentile $6.5 \mathrm{~cm}$ ), circumference was 3.7 inches $(9.31 \mathrm{~cm}$; 5 th percentile $7.75 \mathrm{~cm}$ ), and stretched length was 5.2inches $(13.24 \mathrm{~cm}$; 5 th percentile $10 \mathrm{~cm})$. The erect length was 5.1 inch $(13.12 \mathrm{~cm}$; 5 th percentile $10.25 \mathrm{~cm})$ and erect circumference was 4.5 inches $(11.66 \mathrm{~cm}$; 5 th percentile $9.75 \mathrm{~cm}){ }^{7}$ Stretched penile length correlated highly with erect penile length. Normograms were constructed using these data and provide a useful tool in clinical practice to illustrate the range of variation in penile size. ${ }^{7}$

SPS commonly develops during adolescence when compared among peers is common. ${ }^{2}$ In about 2-thirds of men with SPS, concerns originate during childhood. This has led to the hypothesis that when small boys see others' more developed penises, their own penis looks relatively small. The other third of men with SPS claim their concerns started when they were teenagers after seeing erotic images. In addition, perspective is important: looking down on your own penis instead of looking at it in a mirror makes the penis seem smaller. In addition, being overweight exacerbates the problem. Being bullied or having heard negative remarks about penis size is another reason for insecurity. Obsessive compulsive disorder, social phobia, anxiety, depression, and possibly personality disorders can be comorbid conditions. They also might contribute to the development of penile size dissatisfaction. Sexual dysfunction might be a result of SPS and contributing to it. Finally, insecurity might be a consequence of congenital disorders. ${ }^{6,8}$

\section{ASSESSMENT AND COMORBIDITIES}

A thorough assessment is vital for all men with SPS however they present. As in some cases, their preoccupation might be part of body dysmorphic disorder (BDD) or even psychosis. $\mathrm{BDD}$ is a psychiatric disorder in which a preoccupation with an imaginary imperfection in appearance causes high distress. ${ }^{9,10}$ The person with BDD ruminates about the imperfection, hides the imperfection from others, and regularly checks the imperfection himself. BDD causes impairment in social and occupational functioning and is often accompanied by other mental disorders such as depression. ${ }^{9,10}$

When we as professional therapists meet these men in our practice, we first take an extensive history. This includes a questionnaire to distinguish between those men who have SPS and those with BDD. Veale et $\mathrm{al}^{11}$ developed a validated screening scale to differentiate between the 2 by assessing distress, appearance preoccupation, checking and avoidance behavior, impact on occupation, social life, sexuality, and partner relationship. In our opinion, specific questions around patterns of sexual behavior, expression, and experience are essential to ask. The following topics should be discussed during history taking: sexual orientation, age of first masturbation, age of first sexual intercourse (vaginal or anal), number of sexual partners, relationship duration, pornography use, masturbation frequency, intercourse frequency (vaginal or anal), and avoidance of potential sexual partners or social situations. It is important to verify if current or previous partners are aware of a man's concerns. In addition, a partner's response to these concerns is of importance. Consulting the couple together can be helpful, especially because for most women, penis size is not that important. ${ }^{2,12-14}$

As Veale et $\mathrm{al}^{10}$ reported, there are differences between men with SPS and men with BDD. BDD could be considered a more serious form of SPS with psychiatric comorbidities and in some cases, even delusional aspects. Not all men with SPS have symptoms of BDD. A thorough assessment and diagnosis to differentiate between SPS and BDD is required to determine which treatment is needed.

Not all men with a small penis suffer from SPS. Actually, having a small penis is not a requirement to fulfill the diagnosis of SPS. For example, men who have a micropenis do not fulfill criteria for SPS. They have an objectively small penis, which requires medical treatment and can cause psychological distress. ${ }^{15}$ A micropenis is a congenitally small penis because of "a hormonal abnormality that occurs after the 12th week of gestation." ${ }^{16}$ Micropenis is diagnosed when penile length is 2.5 SDS lower than the mean length for age in combination with a 46XY karyotype and the presence of internal and external genital organs. ${ }^{16}$ Micropenis can also be related to differences of sexual development. ${ }^{15}$ Following this reasoning, not all men requesting penile enhancement treatment can be diagnosed with SPS.

\section{Case Example: John, Part 2, BDD}

In John's case, some characteristics of BDD were identified. He is constantly preoccupied by his size and how this impairs him. He considers himself worthless and unlikely to ever have a partner. He avoids social situations such as the gym or going out with friends to have fun or find a partner. He is distressed, struggles to concentrate at work, and is often absent because of anxiety and low mood. He also experiences erectile dysfunction during masturbation.

Sexual dysfunction and dissatisfaction are related to poor genital self-image and body consciousness. ${ }^{3,17,18}$ Perceived penile inadequacy and an "inability to perform sexually" commonly develop into feelings of shame, hopelessness, and negative selfbelief. This often results in avoidance of peer and sexual or intimate relationships. Distorted cognitions result in inaccurate and idealized beliefs about what partners want sexually, including sweeping generalizations about partner sexuality. The man with SPS typically views potential partners as judgmental, ridiculing, and threatening, which further perpetuates the cognitive rigidity 
and reinforces the belief. Absorption in researching the "problem" and attempting to find a remedy paradoxically promotes and maintains distress. ${ }^{19}$

As is reported in the literature, ${ }^{20}$ most men we encounter in our clinical practice are concerned about flaccid penis size and exhibit severe impairment in their day-to-day functioning. They describe feeling insecure, anxious, and low in mood. They display symptoms of social phobia: often avoiding men's urinals, communal showers, swimwear, and relationships. One author (A.G.) who works in the private sector has also seen an increase in men concerned about erect penis size and who know they are normal but wish to be bigger. This increase may be linked to the accessibility of online sexual images and advances in cosmetic procedures with private clinics offering length and girth enhancement.

\section{SOCIETAL INFLUENCES}

The impact of the internet is pervasive. In the 21st century, comparing oneself with others can occur both online and offline. In our clinical experience, it is less common for men to develop SPS in adulthood. However, when this does occur, it can be directly related to comments by a sexual partner or as the result of a period of distress, depression, or anxiety unrelated to penis size.

\section{Case Example: Karim, Relationship Factors}

Karim (33 years) experienced no concerns during adolescence. The trigger appeared to be related to changes in his sexual relationship with his partner after the birth of their two-year-old child. Before this, he described a frequent and adventurous sexual relationship. However, sexual intimacy had become less frequent, and he felt neglected. Ruminations about penis size began, as did his online research into penile enhancement procedures. He had spoken to his partner who was supportive of his decision to seek enlargement surgery but did not agree that his penis was inadequate. His core belief was that a larger penis would resolve their sexual and intimacy issues.

\section{Case Example: Simon, Mental Problems}

Simon (46 years) had experienced erectile dysfunction since his first sexual experience aged 18 years. His erectile dysfunction resolved when he met his wife aged 23 years who he had married. His decision to marry was in part based on this improvement. After they divorced, he began online dating. Again, he experienced erectile dysfunction. In addition, around this time, his brother died suddenly. Recently, he described his mood as low. He had started to research erectile dysfunction online and began to consider penile enhancement to improve his confidence. His core belief was that a larger penis would resolve his erection difficulties and his low mood.

\section{COUNSELING AND PSYCHOLOGICAL AND PSYCHIATRIC TREATMENT}

Consecutive counseling or treatment always starts with extensive psychoeducation about normal penile size using normograms and pictures. ${ }^{20,21}$ In addition, cognitive therapy is part of the treatment, focusing on recognizing and reframing negative and obstructive cognitions about penis, self, and others. ${ }^{20,21}$ Behavioral interventions are aimed at stopping the avoidance and checking behavior and at improving body image. ${ }^{20}$ In case of depressive or anxious symptoms, selective serotonin reuptake inhibitors can be prescribed. ${ }^{20}$ In more severe cases of BDD or psychotic symptoms, prescribing medication and working together with a psychiatrist is required. ${ }^{20}$ When past experiences are still very influential or even traumatic, eye movement desensitization reprocessing can be useful. The Center for Clinical Interventions in Australia developed a psychological treatment protocol to build body acceptance. This could serve as a clinical guideline for treatment. ${ }^{22}$ These guidelines are referenced and regularly reviewed and updated.

\section{ENHANCEMENT AND SURGERY}

The market for non-invasive penile enhancement devices and strategies has expanded rapidly. Many offer length and girth increase, but robust evidence is often lacking. High levels of distress and reluctance to undergo psychological treatment lead men with SPS to pursue unproven and sometimes dangerous remedies. However, a recent systematic review shows level $2 b$ evidence does exist for the use of penile extenders after surgery in a group of men with normal penis length. ${ }^{23}$ Older studies in a group of men with SPS show level 4 evidence for traction therapy without surgery. Traction therapy offers a length gain with a maximum of approximately $2 \mathrm{~cm} \cdot{ }^{24,25}$ In men with Peyronie's disease, 2 randomized controlled studies show that the use of traction therapy significantly improves stretched penile length with a maximum of $3.0 \mathrm{~cm}{ }^{26,27}$

Besides this, there is a large spectrum of surgical procedures available, such as the division of the suspensory ligament to increase penile length and injection of autologous fat cells, silicone, and hyaluronic acid for girth enhancement. Alongside the usual operative risks, there are specific risks such as paradoxical penile shortening, altered sensation, and postoperative erectile dysfunction. ${ }^{19,28}$ Careful patient selection and a full assessment of patient expectations are essential. ${ }^{19,29}$ Satisfaction rates are low regardless of whether a good surgical outcome is achieved with objective length and or girth gain. ${ }^{19}$ Recently, the protocol and results of using the Penuma silicone sleeve implant were published. ${ }^{30,31}$ Satisfaction with results is high. We speculate this is a result of mainly enhancing circumference in a reliable way, which other techniques until now were not able to do. Dissatisfaction with surgery can result in continually seeking surgical and interventional treatment, or shifting of the problem to another area of the body. This issue is often a reason for insurance claims, so the clinician should carefully explore the expectations of the patient and document it very well.

\section{CONCLUSION AND RECOMMENDATIONS}

SPS is a distressing and invalidating syndrome. Professionals working in urology, andrology, surgery, and sexual medicine should be aware of this syndrome when confronted with a request 
for treatment that might be related. SPS should be taken seriously, and time should be spent on discussing men's concerns and anxieties. In our opinion, medical treatment should not be the first option. Although men with SPS are not easily motivated for psychological treatment, a thorough biopsychosocial assessment is warranted. There is a lot to gain with psychoeducation and psychological interventions, even if surgery is being considered. Psychological interventions can challenge faulty thinking through reframing negative perceptions of size. By bringing an understanding that intimacy is not premised on function and performance alone, self-worth and self-esteem can be developed. These interventions will also contribute to satisfaction with the outcome of surgery. It is clear that working in a multidisciplinary team for assessment and treatment is highly recommended.

\section{OUR RECOMMENDATIONS}

1. Take men with SPS very seriously. They suffer from a great deal of distress and perceive their problem as an anatomical abnormality instead of a psychological issue. This approach is essential to be able to motivate them to consider nonsurgical treatment options.

2. Perform an extensive physical examination to measure objective penis size, excluding any penile abnormality. In addition, detail any previous penile or prostatic surgery which affects penile size or any condition associated with length loss or acquired SPS.

3. All men with SPS should be referred to a specialized psychologist or sexologist for psychological and sexual assessment before deciding which treatment to propose.

4. The most important parts of treatment should be psychoeducation using normograms and pictures.

5. If negative perceptions and cognitions as well as unrealistic expectations are present, counseling or more extensive psychological treatment is required.

6. In cases of BDD or psychosis, psychological treatment combined with prescribing SSRI's and psychiatric consultations is required.

7. A multidisciplinary team is needed to be able to assess the patients request and to decide on the best treatment.

8. The use of penile extender devices (traction therapy) should only be offered to a selected group of highly motivated men who underwent a thorough biopsychosocial assessment and counseling, who cannot be diagnosed with $\mathrm{BDD}$ or other psychiatric disorders, who are very well informed about normal penis size and the expected effect of using penile extender devices, and who still are concerned about their penis length. ${ }^{23}$

9. Preferably do not perform surgical treatment because of the risk of dissatisfaction with the results or shifting of the problem to another area of the penis or body.

10. Surgery should only be performed after thorough biopsychosocial history taking, extensive psychoeducation, and careful exploration of concerns and expectations concerning the result of the surgery.
11. If surgery is performed, this should preferably only be in cases where penile length is 2 SDS lower than the mean, ${ }^{32}$ meaning $<6 \mathrm{~cm}$ flaccid length and $<9.5 \mathrm{~cm}$ stretched flaccid length. ${ }^{7}$ In addition, surgery could be an option when objectively assessed penile abnormalities are present that also impair genital function or sexual performance.

\section{ACKNOWLEDGMENTS}

The authors are grateful to Yacov Reisman, MD, PhD, FECSM, for his critical feedback on the manuscript.

Corresponding Author: Hester Pastoor, MSc, Division of Reproductive Medicine, Department of Obstetrics and Gynecology, Erasmus MC, University Medical Center, dr. Molewaterplein 40, Rotterdam, The Netherlands. Tel: 0031-617048767; Fax: 0031-10-7031753; E-mail: h.pastoor@erasmusmc.nl

Conflict of Interest: The authors report no conflicts of interest.

Funding: None.

\section{STATEMENT OF AUTHORSHIP}

\section{Category 1}

(a) Conception and Design

Hester Pastoor; Angela Gregory

(b) Acquisition of Data

$N / A$

(c) Analysis and Interpretation of Data N/A

\section{Category 2}

(a) Drafting the Article Hester Pastoor; Angela Gregory

(b) Revising It for Intellectual Content Hester Pastoor; Angela Gregory

\section{Category 3}

(a) Final Approval of the Completed Article Hester Pastoor; Angela Gregory

\section{REFERENCES}

1. Murtagh J. The 'small' penis syndrome. Aust Fam Physician 1989;18; 218, 20.

2. Wylie KR, Eardley I. Penile size and the "small penis syndrome". BJU Int 2007;99:1449-1455.

3. Sanches BC, Laranja WW, Alonso JC, et al. Does underestimated penile size impact erectile function in healthy men? Int J Impot Res 2018;30:158-162.

4. Mondaini N, Ponchietti R, Gontero $\mathrm{P}$, et al. Penile length is normal in most men seeking penile lengthening procedures. Int J Impot Res 2002;14:283-286.

5. Veale D, Miles S, Read J, et al. Relationship between selfdiscrepancy and worries about penis size in men with body dysmorphic disorder. Body Image 2016;17:48-56. 
6. Lever J, Frederick DA, Peplau LA. Does size matter? Men's and women's views on penis size across the lifespan. Psychol Men Masculinity 2006;7:129-143.

7. Veale D, Miles S, Bramley S, et al. Am I normal? A systematic review and construction of nomograms for flaccid and erect penis length and circumference in up to 15,521 men. BJU Int 2015;115:978-986.

8. Zachalski W, Krajka K, Matuszewski M. Evaluation of the Treatment of Congenital Penile Curvature Including Psychosexual Assessment. J Sex Med 2015;12:1828-1835.

9. Association AP. Diagnostic and statistical manual of mental disorders, fifth edition. DSM-5 2013.

10. Veale D, Miles S, Read J, et al. Phenomenology of men with body dysmorphic disorder concerning penis size compared to men anxious about their penis size and to men without concerns: a cohort study. Body Image 2015;13:53-61.

11. Veale D, Miles S, Read J, et al. Penile Dysmorphic Disorder: Development of a Screening Scale. Arch Sex Behav 2015; 44:2311-2321.

12. Eisenman R. Penis size: Survey of female perceptions of sexual satisfaction. BMC Womens Health 2001;:1.

13. Francken $A B$, van de Wiel HBM, van Driel MF, et al. What importance do women attribute to the size of the penis? Eur Urol 2002;42:426-431.

14. Stulhofer A. How (un)important is penis size for women with heterosexual experience? Arch Sex Behav 2006;35:5-6.

15. Kayes O, Shabbir M, Ralph D, et al. Therapeutic strategies for patients with micropenis or penile dysmorphic disorder. Nat Rev Urol 2012;9:499-507.

16. Hatipoglu N, Kurtoglu S. Micropenis: etiology, diagnosis and treatment approaches. J Clin Res Pediatr Endocrinol 2013; 5:217-223.

17. van den Brink F, Vollmann M, Sternheim LC, et al. Negative Body Attitudes and Sexual Dissatisfaction in Men: The Mediating Role of Body Self-Consciousness During Physical Intimacy. Arch Sex Behav 2018;47:693-701.

18. Veale D, Miles S, Read J, et al. Sexual Functioning and Behavior of Men with Body Dysmorphic Disorder Concerning Penis Size Compared with Men Anxious about Penis Size and with Controls: A Cohort Study. Sex Med-Uk 2015;3:147-155.

19. Kayes $O$, Chandra $L$, Yates A. Micropenis and penile dysmorphobia: diagnosis, management, outcomes, and future developments. In: Minhas SM JP, ed. Male sexual dysfunction: a clinical guide. Wiley Blackwell; 2016. p. 236-245.
20. Ghanem H, Glina S, Assalian P, et al. Position paper: Management of men complaining of a small penis despite an actually normal size. J Sex Med 2013;10:294-303.

21. Ghanem H, Shamloul R, Khodeir F, et al. Structured management and counseling for patients with a complaint of a small penis. J Sex Med 2007;4:1322-1327.

22. Interventions CfC. (2018) Building Body Acceptance. [www document]. Available at: https://www.cci.health.wa. gov.au/Resources/Looking-After-Yourself/Body-Dysm orphia. Accessed August 23, 2019.

23. Marra G, Drury A, Tran L, et al. Systematic Review of Surgical and Nonsurgical Interventions in Normal Men Complaining of Small Penis Size. Sex Med Rev 2020;8:158-180.

24. Nikoobakht M, Shahnazari A, Rezaeidanesh M, et al. Effect of penile-extender device in increasing penile size in men with shortened penis: preliminary results. J Sex Med 2011;8:31883192.

25. Nowroozi MR, Amini E, Ayati M, et al. Applying extender devices in patients with penile dysmorphophobia: assessment of tolerability, efficacy, and impact on erectile function. J Sex Med 2015;12:1242-1247.

26. Moncada I, Krishnappa P, Romero J, et al. Penile traction therapy with the new device 'Penimaster PRO' is effective and safe in the stable phase of Peyronie's disease: a controlled multicentre study. BJU Int 2019;123:694-702.

27. Ziegelmann M, Savage J, Toussi A, et al. Outcomes of a Novel Penile Traction Device in Men with Peyronie's Disease: A Randomized, Single-Blind, Controlled Trial. J Urol 2019; 202:599-610.

28. Furr J, Hebert K, Wisenbaugh E, et al. Complications of Genital Enlargement Surgery. J Sex Med 2018;15:1811-1817.

29. Littara A, Melone R, Morales-Medina JC, et al. Cosmetic penile enhancement surgery: a 3 -year single-centre retrospective clinical evaluation of 355 cases. Sci Rep 2019;9:6323.

30. Elist JJ, Levine L, Wang R, et al. Patient selection protocol for the Penuma(R) implant: suggested preoperative evaluation for aesthetic surgery of the penis. Int J Impot Res 2020;32:149152.

31. Elist JJ, Valenzuela R, Hillelsohn J, et al. A Single-Surgeon Retrospective and Preliminary Evaluation of the Safety and Effectiveness of the Penuma Silicone Sleeve Implant for Elective Cosmetic Correction of the Flaccid Penis. J Sex Med 2018;15:1216-1223.

32. Wessells H, Lue TF, McAninch JW. Penile length in the flaccid and erect states: guidelines for penile augmentation. J Urol 1996;156:995-997. 